\title{
Considerations over a Case of Suspected Therapeutic Failure in Pediatric Patients after Switching Valproate Manufacturers
}

\author{
Jaqueline Kalleian Eserian, PhD ${ }^{1,2}$; Jair Ribeiro Chagas, $P h D^{1}$; José Carlos Fernandes Galduróz, PhD \\ ${ }^{1}$ Departamento de Psicobiologia, Universidade Federal de São Paulo. São Paulo, Brazil \\ ${ }^{2}$ Centro de Medicamentos, Cosméticos e Saneantes, Instituto Adolfo Lutz. São Paulo, Brazil
}

\begin{abstract}
Introduction: Product switching followed by suspected adverse events are common and unsettling for antiepileptic drugs. The objective of this case study was to describe the investigation performed after report of suspected therapeutic failure in pediatric patients following a switch to a different valproate manufacturer and identify strategies concerning medication management for improving therapeutic outcomes. Case description: It was reported that different pediatric patients' condition changed (agitation/ seizures) after refilling the same drug prescription (sodium valproate syrup) from a different manufacturer. Medical staff reported a suspected therapeutic failure and some units of the product batch associated with the problem were seized by the local Post-marketing Surveillance Service for investigation of potential quality deviations. The seized units were evaluated by the State's Surveillance Laboratory, nevertheless, drug potency was found to be $98.7 \%$. Conclusion: We consider that the reported event could be associated with aspects of medication use, i.e. potential dose measurement deviations resulting from remaining of residual liquid in the cup or eventual delay at prescription refilling process and consequential - even though brief - pharmacotherapy discontinuity. Patient education and counseling by pharmacists are essential for preventing drug-related problems and enhancing positive outcomes of pharmacotherapy.
\end{abstract}

Keywords: suspected therapeutic failure, pediatric patients, valproate, medication management, pharmacist

\section{Introduction}

Liquid medications facilitate drug administration for pediatric patients and allow dose adjustment ${ }^{1}$ - an essential part of neuropsychiatric drugs management. Nevertheless, measuring devices used for drug delivery are associated with a potential risk of under or overdosing, ${ }^{2}$ which is particularly critical for narrow therapeutic index (NTI) drugs such as valproate.

Antiepileptic drugs (AED) switching is referred to the change from one product to another of the same drug, i.e., brand to generic, generic to brand, or generic to generic AED products from different manufacturers.,

Some studies have speculated that small variations in bioavailability between products - including those within the standard bioequivalence range - could result in adverse clinical outcomes when patients switch between manufacturers of a NTI drug, such as AED. ${ }^{5}$

Concern over this topic is supported by reports of product switching followed by suspected adverse events, which are particularly common for AED and have been extensively discussed because of their unexpected clinical outcomes such as loss of seizure control, increased seizure frequency, occurrence of adverse drug effects, hospitalization and higher healthcare services use. ${ }^{3-6}$

Corresponding author: José Carlos Fernandes Galduróz, PhD Rua Napoleão de Barros, 925, CEP 04024-002, São Paulo, SP, Brazil; Phone number: +55 1121490168

Fax number: +55 11 21490155; Email: galduroz@unifesp.br
Different observational studies have been conducted to identify potential links between AED switching and seizure related events, however, most of the studies were subject to bias and general results were conflicting. 7,8

The objective of this case study was to describe the investigation performed after report of suspected therapeutic failure in pediatric patients following a switch to a different valproate manufacturer and identify strategies concerning medication management for improving therapeutic outcomes.

\section{Case description}

The mother of a pediatric patient under treatment in a specialties public outpatient facility noted that her son's condition changed after refilling the same drug prescription $250 \mathrm{mg} / 5 \mathrm{~mL}$ sodium valproate syrup packaged in plastic bottles with accompanying dosing cup - from a different manufacturer. Doses were measured with the dosing cup packaged with the medication. Investigation pointed that other patients' mothers from the same outpatient facility also noticed that their children became agitated or even had seizures after switching between the products. Each patient was put on the same two manufacturers of valproate syrup in the same sequence, as medication was dispensed by the outpatient facility systematically. No information regarding dose changing or any other factor that could have influenced patients' condition was reported. Accordingly, medical staff reported a suspected therapeutic failure.

Some units of the product batch associated with the problem were seized by the local Post-marketing Surveillance Service for 
investigation. The seized units were evaluated by the State's Surveillance Laboratory. Potential deviations in drug potency were evaluated using a HPLC-DAD system (Waters, Milford, USA) at the following conditions: $\mathrm{C} 8$ column $(4.0 \times 250 \mathrm{~mm}$, $5 \mu \mathrm{m}$, Agilent, Santa Clara, USA), flow rate of $1 \mathrm{~mL} / \mathrm{min}$, injection volume of $20 \mu \mathrm{L}$ and detection set at $215 \mathrm{~nm}$. Mobile phase consisted of monobasic sodium phosphate buffer solution (Vetec/Sigma-Aldrich, Duque de Caxias, Brazil) and acetonitrile (Merck, Darmstadt, Germany). Valproic acid reference standard (USP, Rockville, USA) was used for quantitation of the sample. The amount of sodium valproate was expressed as valproic acid. ${ }^{9}$

Results showed that the product contained $98.7 \%$ of the labeled amount of valproic acid (specification: $90-110 \%{ }^{9}$ ). The amount of drug in individual doses could not be checked as there is no pharmacopeial test for dose delivery using dosing cups.

\section{Discussion}

As a NTI drug, a small variation in valproate dose might result in serious therapeutic failure or adverse reactions. ${ }^{10}$ Also, pediatric patients usually require a weight-based dosing strategy, thus a small variation in the measured dose may also have clinical implications, ${ }^{11}$ requiring increased attention.

The laboratory analysis performed on the seized units showed that the reported suspected therapeutic failure was not associated with any deviation in drug product potency. Thus, we discuss three different hypotheses regarding the medication for elucidating the case:

\section{A. Inaccurate measurement of doses}

High rates of inaccuracy in doses measured with dosing cups have been reported previously. ${ }^{1,12,13}$ Sobhani et al, 2008 found that only $14.6 \%$ of participants measured an acceptable dose ( $\pm 10 \%$ of the requested volume) of $5 \mathrm{~mL}$ of acetaminophen suspension when using the cup, with a measured mean volume of $6.3 \pm 0.7 \mathrm{~mL}$. Only $4.2 \%$ of the participants measured an accurate dose $(5 \mathrm{~mL})$ when using the cup. Also, they were five times less likely to measure acceptable doses when using the cup versus the dosing syringe. ${ }^{13}$

Caregivers of a minor patient who has been taking sodium valproate syrup for a long time are expected to measure the prescribed doses accurately. Nevertheless, dosing cups potentially retain residual liquid after dose administration, especially when measuring viscous formulations. ${ }^{14}$ Supposing that the refilled new formulation was significantly more viscous than the original one, a potential unobserved remaining of residual liquid in the cup would result in the delivery of underdoses with consequent clinical outcomes.

Abu-Geras et al, 2016 found that accuracy decreased for cup measurements when viscosity of the liquid increased. In contrast, the measurement of higher viscosity liquids was more accurate when using a dosing syringe. ${ }^{15}$

\section{B. Variability between manufacturers of the same drug}

The standard bioequivalence range of $80-125 \%$ has been pointed by some as too broad to avoid clinical differences in bioavailability between different products, especially for NTI drugs, such as AED. ${ }^{7}$

It has been suggested that AED bioequivalence may not demonstrate therapeutic equivalence between products, i.e., variability within accepted limits would still compromise seizure control. ${ }^{4}$

Theoretically, the difference between two generic products would be even greater, as they could fall at extremes of the brand product's bioequivalence range. ${ }^{7,16}$

In 2016, the American Epilepsy Society stated that substitution with generic products approved by the FDA reduces treatment costs and does not compromise its efficacy. ${ }^{16,17}$ Indeed, generic drugs have been associated with better adherence than their corresponding brand products due to low costs. Enhancing patient adherence is essential for treatment with AED, considering that greater medication adherence contributes for achieving and maintaining effective blood levels and consequently results in better clinical outcomes. ${ }^{7}$

Nevertheless, adverse outcomes associated with AED switching still concern patients and clinicians. Indeed, some authors recommend that whenever possible patients should be kept on a single AED manufacturer to avoid product switching. 8,18

Conversely - because of the dosage form - drug release from the drug product is assumed to be self-evident for syrups and other solubilized forms. Accordingly, if the drug product also contains the active ingredient in the same concentration and dosage form as the reference listed drug and does not contain excipients or changes in formulation that significantly affects drug absorption or availability, in vivo bioavailability data can be waived. ${ }^{19-21}$

Considering that valproate products were given as syrup, it is unlikely that the suspected therapeutic failure reported in this case was associated with any bioavailability differences between manufacturers.

\section{Delay in refilling or variation in drug use pattern}

Association between interchangeability and seizure events might result to some extent from delay in refilling, lack of information about the medication, difficulties in managing a complex therapeutic regimen, temporary treatment nonadherence or small alteration in the medication use pattern. ${ }^{5}$

Some studies pointed that prescription refilling itself - even when involving the same medicine from the same manufacturer at the same strength - was associated with an elevated risk of seizure-related events. ${ }^{5,16}$ 
Patients were switched among different manufacturers because the facility's pharmacy changed its supply source. An eventual delay during restocking would potentially have uncovered refilling process and might then be identified as the common cause for the reported event, as therapeutic failure was reported during the exact same period for more than one patient.

Investigation was focused on aspects regarding the product as Post-marketing Surveillance Service initially suspected that the reported event was a result of quality deviation in the potency of the medication. However, potency was proven to be within the acceptable limits after laboratory tests and this matter was discarded.

Limitations of this investigation include that specific details related to the case (patients' medical and refill records, serum concentrations of the drug, investigation of poor/ fast metabolizers and exact number of patients) were not described in the report form that went with the seized product. Also, we could not compare viscosity measurements as the original formulation used by the patients was not seized for laboratory tests.

It is crucial to implement and continue operating functional prescription refilling systems. Even when facing an eventual manufacturer switching during the medication purchase process, prescription refilling should not be uncovered, especially for neuropsychiatric drugs and others which demand continuous use.

Patients might be assisted for selecting the best device for measuring the liquid medication according to their needs, considering product's own characteristics. Using a dosing syringe instead of a cup might be more appropriate for measuring doses of viscous formulations containing NTI drugs.

The management of the prescribed therapeutic regimen should be reinforced by pharmacists in order to avoid patients skipping doses or changing medication use instructions, e.g. timing, dosing and duration of treatment, which potentially would affect its efficacy.

Indeed, it has been suggested that alteration in medication use pattern is just as important as pharmacokinetics regarding variation in clinical response. ${ }^{5}$

\section{Conclusion}

Aspects of medication use may have contributed for the assumed association between manufacturer switching and loss of seizure control in pediatric patients. We consider that the reported therapeutic failure could be associated with potential dose measurement deviations resulting from remaining of residual liquid in the cup or eventual delay at prescription refilling process and consequential - even though brief pharmacotherapy discontinuity.
Further investigations regarding caregivers' ability to measure prescribed doses accurately are needed. Also, different aspects of AED switching still need to be investigated, as findings remain conflicting.

We recommend the use of a dosing syringe instead of a dosing cup for dose measurements of critical drugs, such as valproate. Still, we suggest that other factors such as medication adherence and variation in drug use pattern be considered when investigating associations between AED switching and reduced clinical response.

Patient education and counseling by pharmacists are essential for preventing drug-related problems and enhancing positive outcomes of pharmacotherapy.

Conflicts of Interest: We declare no conflicts of interest or financial interests that the authors or members of their immediate families have in any product or service discussed in the manuscript, including grants (pending or received), employment, gifts, stock holdings or options, honoraria, consultancies, expert testimony, patents and royalties.

\section{References}

1. Ryu GS, Lee YJ. Analysis of liquid medication dose errors made by patients and caregivers using alternative measuring devices. J Manag Care Pharm. 2012;18:439-445.

2. Food and Drug Administration. Dosage Delivery Devices for Orally Ingested OTC Liquid Drug Products. Guidance for Industry; 2011. Available at: https://www.fda.gov/media/78087/download. Accessed 14 Mar 2017.

3. Kesselheim AS, Stedman MR, Bubrick EJ, et al. Seizure outcomes following the use of generic versus brandname antiepileptic drugs: a systematic review and meta-analysis. Drugs. 2010;70:605-621.

4. Kwan P, Palmini A. Association between switching antiepileptic drug products and healthcare utilization: A systematic review. Epilepsy Behav. 2017;73:166172.

5. Gagne JJ, Avorn J, Shrank WH, et al. Refilling and switching of antiepileptic drugs and seizure-related events. Clin Pharmacol Ther. 2010;88:347-353.

6. Erickson SC, Le L, Ramsey SD, et al. Clinical and pharmacy utilization outcomes with brand to generic antiepileptic switches in patients with epilepsy. Epilepsia. 2011;52:1365-1371.

7. Gagne JJ, Kesselheim AS, Choudhry NK, et al. Comparative effectiveness of generic versus brandname antiepileptic medications. Epilepsy Behav. 2015;52:14-18.

8. Perucca E. The safety of generic substitution in epilepsy. Lancet Neurol. 2016;15(4):344-345. 
9. United States Pharmacopoeia. US Pharmacopoeial Convention, $39^{\text {th }}$ ed. Rockville: US Pharmacopoeial Convention; 2016.

10. Sarpatwari A, Lee MP, Gagne JJ, et al. Generic versions of narrow therapeutic index drugs: A national survey of pharmacists' substitution beliefs and practices. Clin Pharmacol Ther. 2018;103:1093-1099.

11. Wojewoda E, Chou KJ. Factors associated with dispensing dosage delivery devices. J Pediatr Pharmacol Ther. 2017;22:251-255.

12. Yin HS, Mendelsohn AL, Wolf MS, et al. Parents' medication administration errors: role of dosing instruments and health literacy. Arch Pediatr Adolesc Med. 2010;164:181-186.

13. Sobhani P, Christopherson J, Ambrose PJ, et al. Accuracy of oral liquid measuring devices: comparison of dosing cup and oral dosing syringe. Ann Pharmacother. 2008;42:46-52.

14. Wening K, Breitkreutz J. Oral drug delivery in personalized medicine: unmet needs and novel approaches. Int J Pharm. 2011;404:1-9.

15. Abu-Geras D, Hadziomerovic D, Leau A, et al. Accuracy of tablet splitting and liquid measurements: an examination of who, what and how. J Pharm Pharmacol. 2017;69:603-612.
16. Kesselheim AS, Bykov K, Gagne JJ, et al. Switching generic antiepileptic drug manufacturer not linked to seizures: A case-crossover study. Neurology. 2016;87:1796-1801.

17. American Epilepsy Society. Substitution of Different Formulations of Antiepileptic Drugs for the Treatment of Epilepsy; 2016. Available at:

https://www.aesnet.org/about_aes/generic-positionstatement. Accessed 02 Feb 2019.

18. Privitera M. Generic substitution of antiepileptic drugs: What's a clinician to do? Neurol Clin Pract. 2013;3(2):161-164.

19. Food and Drug Administration. Code of Federal Regulations. Title 21, Volume 5, 21 CFR 320.22(b)(3). Criteria for waiver of evidence of in vivo bioavailability or bioequivalence. 2019. Available at: https://www.accessdata.fda.gov/scripts/cdrh/cfdocs/ cfcfr/CFRSearch.cfm?fr=320.22. Accessed 05 Dec 2019.

20. Food and Drug Administration. Bioavailability Studies Submitted in NDAs or INDs - General Considerations. Guidance for Industry; 2019. Available at: https://www.fda.gov/media/121311/download. Accessed 12 Dec 2019.

21. Niazi, SK. Handbook of Bioequivalence Testing. Drugs and Pharmaceutical Sciences, $2^{\text {nd }}$ ed. Boca Raton: CRC Press; 2015. 